\title{
Case Report \\ A Case Report of Rectorrhagia After Improved Heavy Menstrual Bleeding and Investigation of its Cause Based on Traditional Persian Medicine
}

\author{
Atieh Sadat Danesh ${ }^{1,2}, *$ Fatemeh Nojavan ${ }^{1,3}$ (1)
}

1. Department of Traditional Persian Medicine, School of Traditional Persian Medicine, Qom University of Medical Sciences, Qom, Iran

2. Student Research Committee, Qom University of Medical Sciences, Qom, Iran.

3. Spiritual Health Committee, Qom University of Medical Sciences, Qom, Iran.

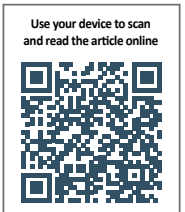

Clttation: Danesh AS, Nojavan F. [A Case Report of Rectorrhagia After Improved Heavy Menstrual Bleeding and Investigation of its Cause Based on Traditional Persian Medicine (Persian)]. Journal of Arak University of Medical Sciences (JAMS). 2020; 23(1):118-125. https://doi.org/10.32598/JAMS.23.1.5968.1

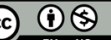

Article Info:

Received: 13 Aug 2019

Accepted: 05 Mar 2020

Available Online: 01 Apr 2020

Key words:

Hemorrhoidal bleeding, Heavy menstrual bleeding, Iranian traditional medicine, Transmissible diseases

\section{ABSTRACT}

Background and Aim From the viewpoint of Iranian Traditional Medicine (ITM), hemorrhoidal bleeding is not only an organic disease; it also indicates the quality and quantity of blood and temperaments in the body. According to this view, bleeding from different areas has several causes, two important causes of which are: a. vascular hyperemia and consequent blooding in bleeding-prone areas, and b. changes in blood quality that cause the arteries to open and bleed. This study, by reporting a case of hemorrhoidal bleeding, is an evidence of some therapeutic principles in ITM.

Case Report Patient was a 39-year-old married woman with four children and warm-wet temperament complaining of heavy menstrual bleeding for one year. After three months of herbal drug administration, menstrual bleeding became normal. One month later, she had hemorrhoidal bleeding. Her bleeding was treated based on ITM method by removing the black bile (Soda) from the body, phlebotomy and leech therapy around the anus.

Ethical Considerations This study has been approved by the Research Ethics Committee of Arak University of Medical Sciences with code: IR.MUQ.REC.1396.110.

Conclusion Based on ITM, it seems that the cause of hemorrhoidal bleeding in this case is vascular hyperemia and poor blood quality following symptomatic treatment of heavy menstrual bleeding.

\section{Extended Abstract}

\section{Introduction}

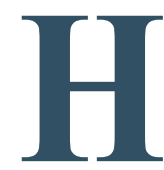

emorrhoids, resulted from the dilation of veins in the submucosal layer of the lower rectum, are a common cause of lower gastrointestinal bleeding $[1,2]$. In common medicine, the main cause of hemorrhoids is weakness of the anal connective tissue and increased pressure inside the arteriovenous anastomoses, which may bleed due to fecal excretion and increased pressure [3], while from the point of view of Iranian Traditional Medicine (ITM), hemorrhoidal bleeding is not only a organic disease, but also indicates the quality and quantity of blood and temperaments in the body. According to this view, bleeding from different parts of the body has different causes, two important causes of which are: A. vascular hyperemia and consequent bleeding in bleeding-prone areas; and B. change in blood quality

\section{* Corresponding Author:}

Fatemeh Nojavan, PhD.

Address: Department of Traditional Persian Medicine, Panzdahekhordad Blvd, Qom, Iran.

Tel: +98 (914) 3537460

E-mail: fnojavan@muq.ac.ir 
that causes the arteries to open and bleed [8-14]. This study, by reporting a case of hemorrhoidal bleeding, is evidence of a series of therapeutic principles in ITM.

\section{Case Report}

The patient was a 39-year-old married woman with five children complaining of heavy menstrual bleeding for a year. The patient did not take any special medication. Her blood pressure was 180/80 $\mathrm{mm} \mathrm{Hg}$, with occasional increases in blood pressure. She noted the history of hemorrhoidal bleeding before severe menstrual bleeding. According to the diagnosis of an ITM specialist, the patient's temperament was warm and wet. In order to control menstrual bleeding for three months, symptomatic treatment was performed without considering the main cause of bleeding by using one of the herbal medicines for hemorrhage called "Shepherd's Purse".

After three months of medication, menstrual bleeding returned to normal state. One month after healing of menstrual bleeding, the patient experienced clear bleeding from the anus without a history of trauma to the hemorrhoid site or severe constipation following defecation and anal pain. On examination, several swollen and non-thrombotic external hemorrhoids were observed. According to a general surgeon, no surgery was needed. According to an ITM specialist, the patient was given an oral medication for her melancholic temperament, phlebotomy and leech therapy around the anus for a month, which relieved the pain and bleeding and reduced the hemorrhoids.

\section{Results}

Hyperemia-related diseases, including those expressed in ITM, are caused by changes in the quantity and quality of temperaments in the body [16]. Increasing the volume of fillers in body spaces can cause these spaces to stretch and tear. Arteries are one of these spaces that rupture and bleed if the volume and blood pressure increase in low-resistance areas. According to ITM, blood is composed of four humors, and any change in their quality and quantity can cause disease. One of these types is melancholy humor. According to the principles of ITM, the cause of hemorrhoids is an increase in abnormal melancholic temperament. Blood containing abnormal melancholic temperament tends to be excreted from different parts of the body during circulation. Substances with lower concentrations and higher sharpness cause bleeding in various places such as the nose, by stimulating and contaminating the walls of the arteries.

More concentrated substances cannot pass through the arterial pores and tend to the lower parts of the body, causing swelling or bleeding in susceptible areas such as the uterus and anus [11, 17]. In ITM, it is emphasized that bleeding from these areas prevents many diseases and should not be stopped suddenly [10]. If prevented, other parts of the body will get sick. In other words, some diseases can be cured by shifting to another disease. For example, menstrual bleeding is beneficial for a patient with hemorrhoids, because the blood is expelled from the uterus instead of hemorrhoids, and on the other hand, rectal bleeding is beneficial for uterine diseases [18].

In the study patient, there were both signs of vascular hyperemia and increased blood amount, as well as signs of a change in its quality. Both of these factors contributed to the patient's heavy menstrual bleeding. By stopping menstrual bleeding with medication, the blood tends to come out of a duct that was previously prone to bleeding. The presence of hemorrhoidal vessels in this patient is a protective factor against damage caused by vascular hyperemia and high blood pressure. It prevents the elimination of substances cause melancholic temperament, and is also able to relieve menstrual bleeding symptoms. In the study patient, by regulating the quality and quantity of blood, phlebotomy (Fasd) controlled both hemorrhoidal and menstrual bleeding.

The main purpose of this study is not to express the method of hemorrhoid treatment based on ITM, but to suggest the cleansing of the body for radical treatment of many diseases by indicating one of the most common medical errors, i.e. symptomatic treatment of the disease.

\section{Discussion}

In treatment based on the principles of ITM, eliminating the main cause of the disease is a priority over eliminating the complications of the disease. It may trigger another disease by eliminating the complications of one disease. On the other hand, having one disease makes one immune to another disease. Therefore, paying close attention to the participation of members in the development of the disease and the relationship between the diseases are of great importance in treatment. It is recommended that it is not enough just to eliminate the symptoms in the treatment of diseases, but also, by considering the main cause of the disease, the relationship between the symptoms created in one organ and the development or prevention of the disease in other organs should be taken into account. 


\section{Ethical Considerations}

Compliance with ethical guidelines

This study has been approved by the research ethics committee of Arak University of Medical Sciences with Code: IR.MUQ.REC.1396.110.

\section{Funding}

This research did not receive any specific grant from funding agencies in the public, commercial, or not-forprofit sectors.

Authors' contributions

All authors met the writing standards based on the recommendations of the International Committee of Medical Journal Editors (ICMJE).

Conflicts of interest

The authors declare no conflict of interest.

Acknowledgements

The authors would like to thank the School of Traditional Iranian Medicine, Qom University of Medical Sciences for their cooperation. 


\title{
كَزارش يك مورد ركتورأزى بعد از بهبود خونريزى شديد رحمى و بررسى علت آن بر اساس مبانى طب سنتى ايرانى نورد
}

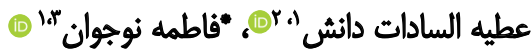 \\ ا ا. كروه طب سنتى ايرانى، دانشكده طب سنتى ايرانى، دانشعاه علوم يزشكى قهم، قهه ايران.

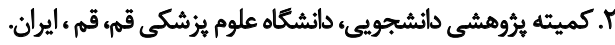

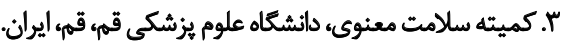

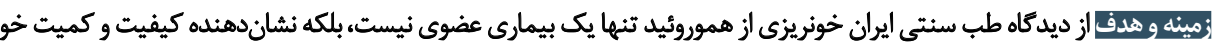

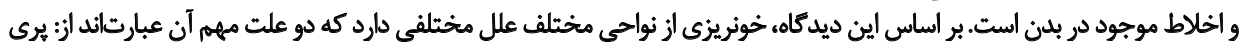

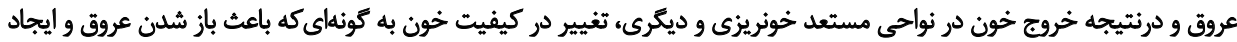

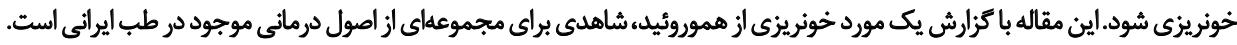

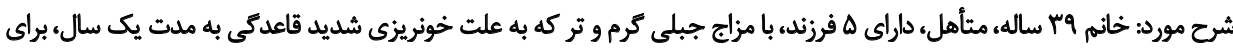

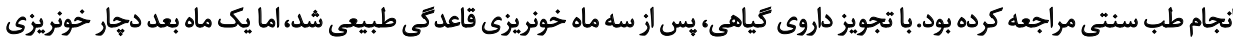

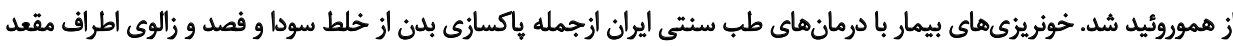

البهبود يبيداكرد.

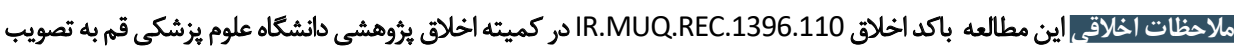

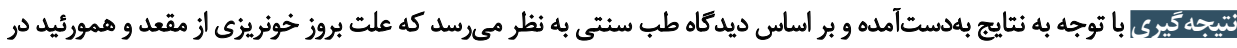

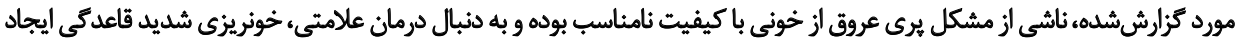

كليدوأرهاء

خونريزى هموروئيد،

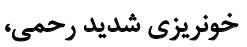

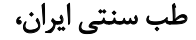

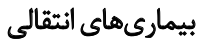

شدهاند، ممكن است با درد و ترومبوز بروز كنند؛ باوجوداين بين

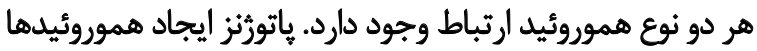

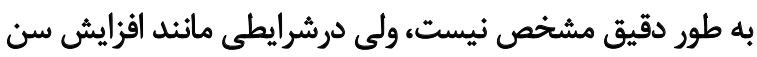

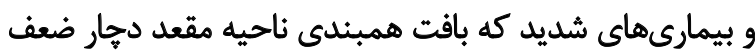

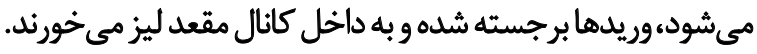

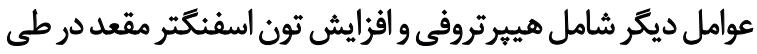

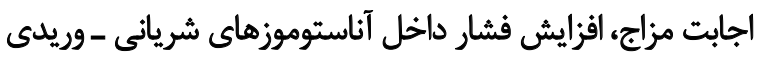

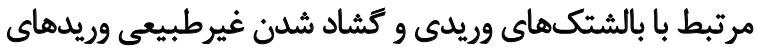

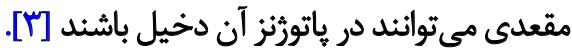

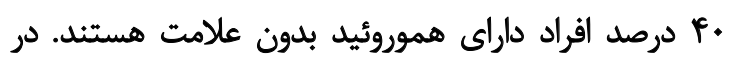

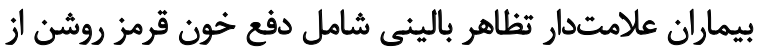

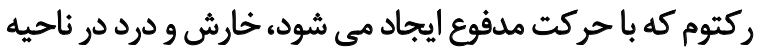

مقعد همراه با هموروئيدهاى ترومبوزه است [ن]

مقدمه

هموروئيدها يكى از شايعترين علل خونريزى از دستخاه كوارش

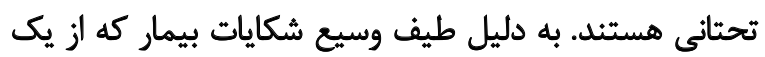

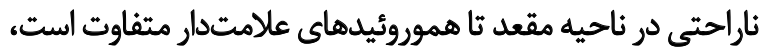

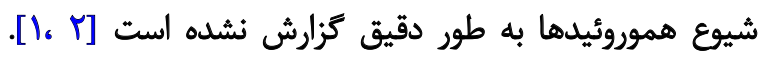

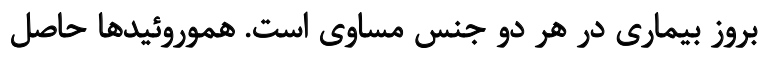

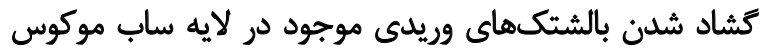

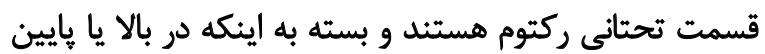

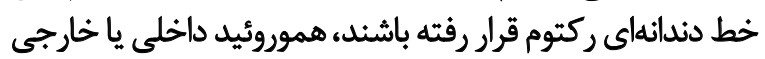

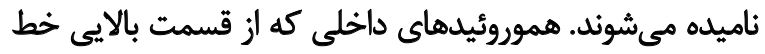

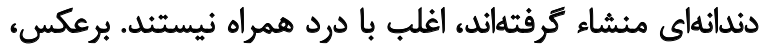

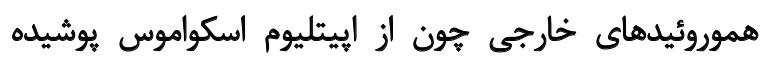

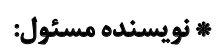

فاطمه نوجوان

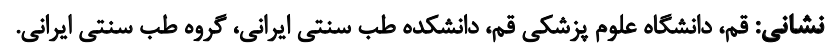

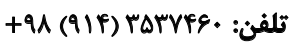
يست الكترونيكى: fnojavan@muq.ac.ir 


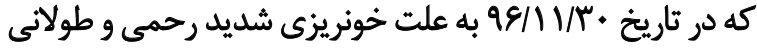

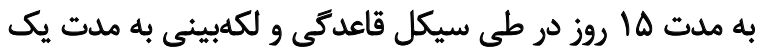

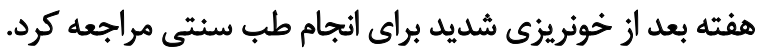

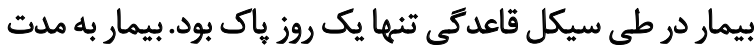

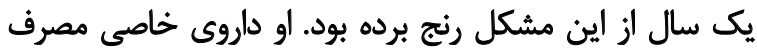

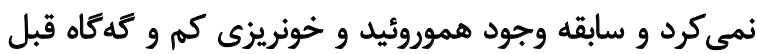

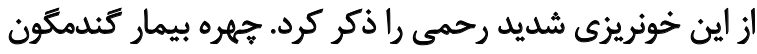

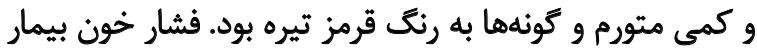

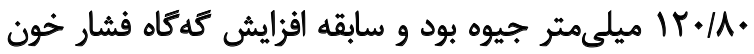

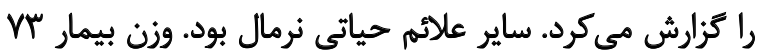

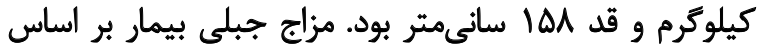

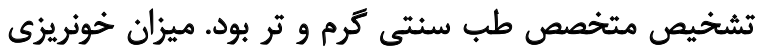

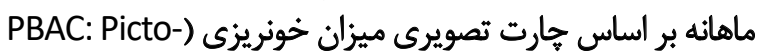

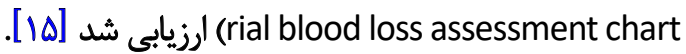
در اين جارت كه بر اساس تعداد يد بهداشتى و ميزان

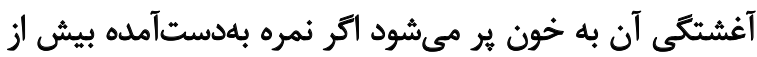

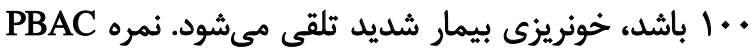

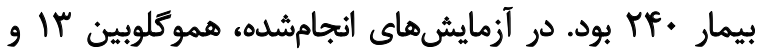

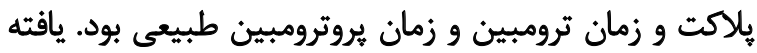

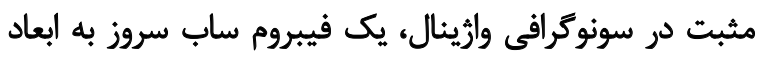

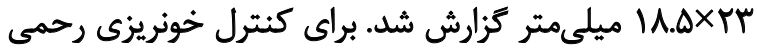

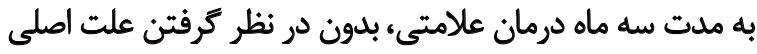

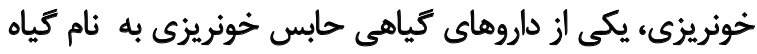

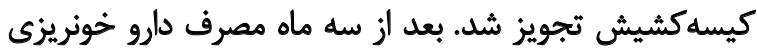

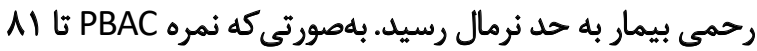

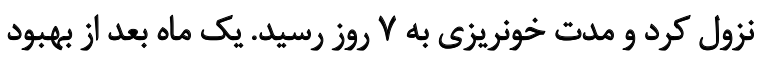

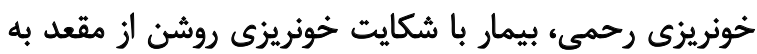

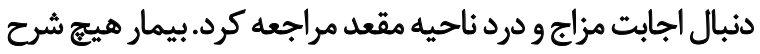

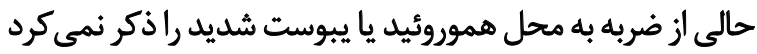

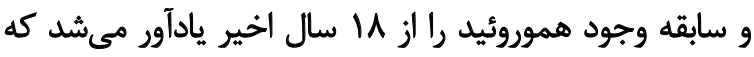

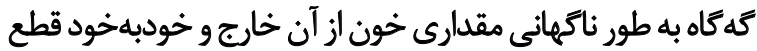

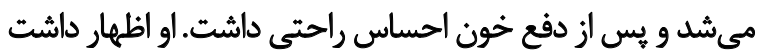

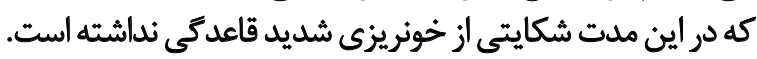

در يك سال اخير نيز دفع خون از مقعد نداشته، ولى مبتلا بـ مدابه

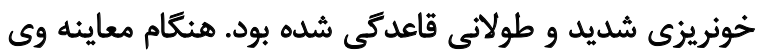

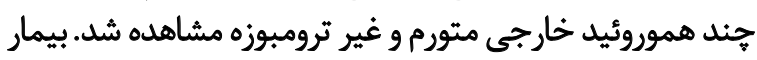

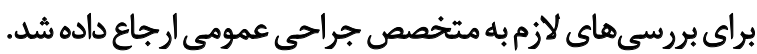

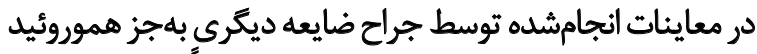

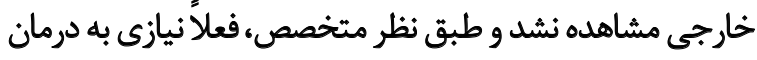

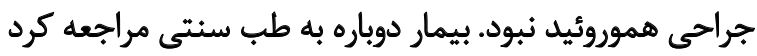

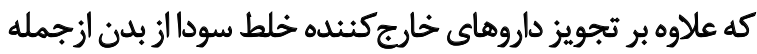

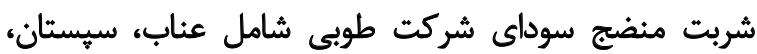

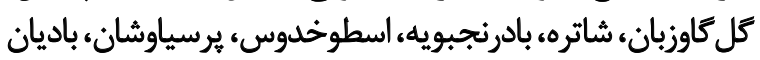

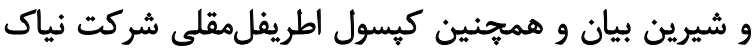

راههاى تشخيصى عبارتاند از مشاهده مستقيم هموروئيدها،

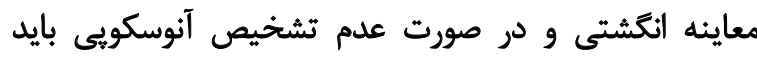

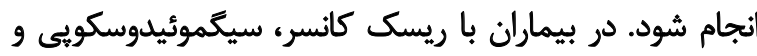
كولونوسكويى بايد انجام يذيرد [هان].

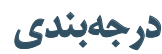

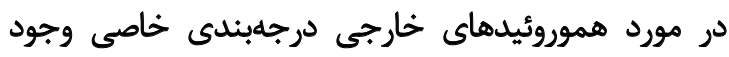

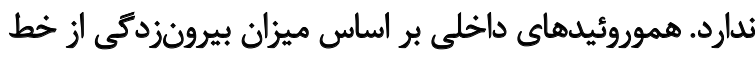

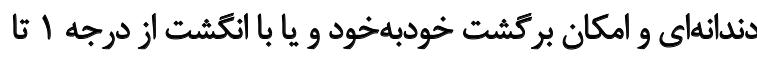

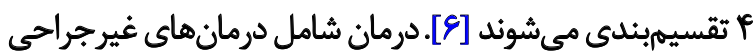

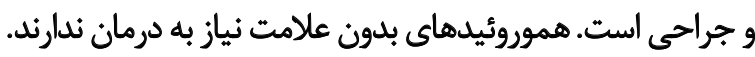

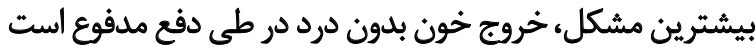

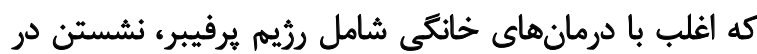

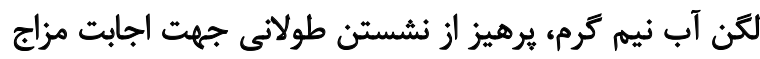

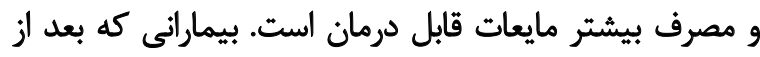

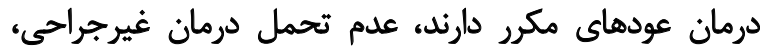

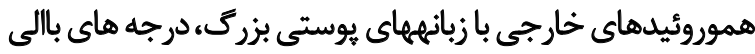

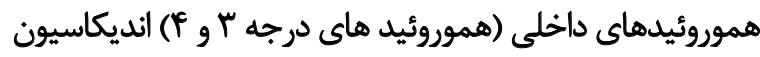

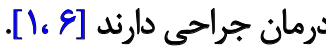

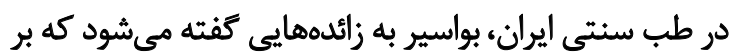

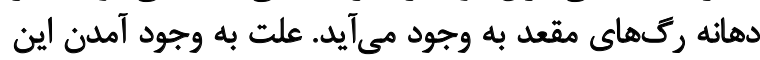

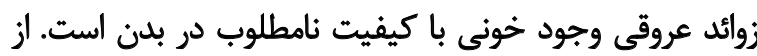

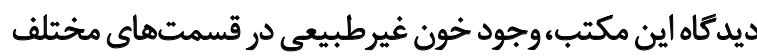

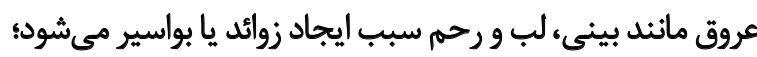

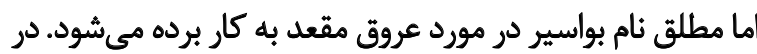

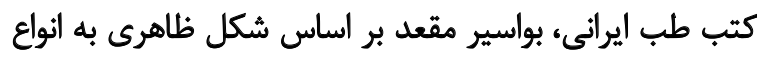

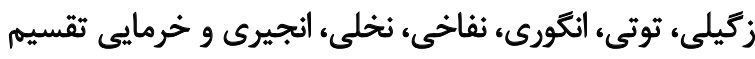

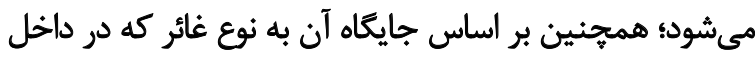

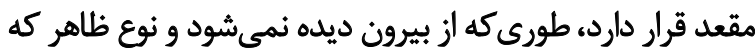

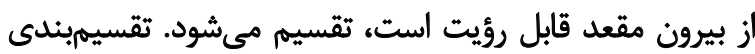

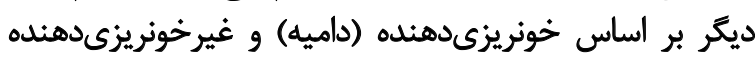

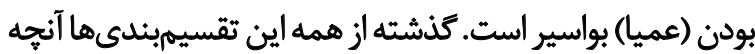

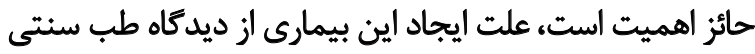

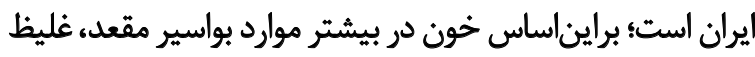

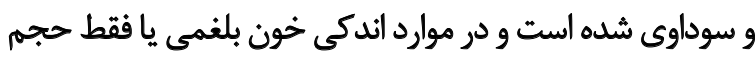

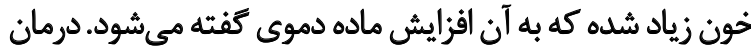

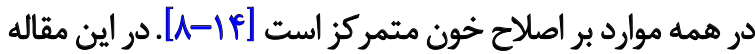

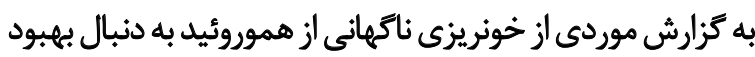

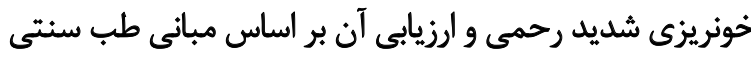
ايران يرداخته شده است. كزارش مورد خانم وج ساله، متأهل و داراى ه فرزند با زايمانهاى طبيعى بود 
(افزايش حجم يا فشار خون) يا حركت و فرياد قوى ذكر كردهاند.

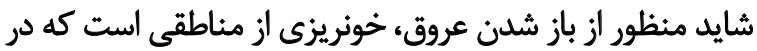

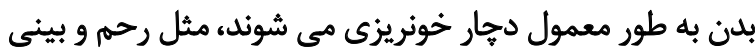

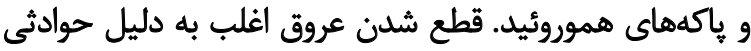

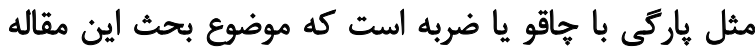

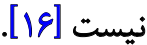

علامت كسى كه به خاطر امتلاء عروق دجار خونريزى مي جيود،

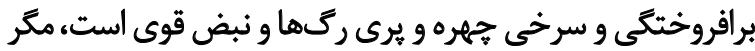

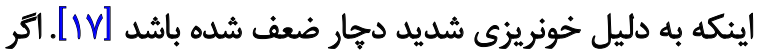

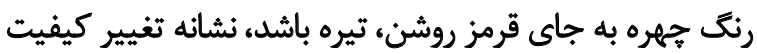

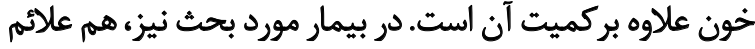

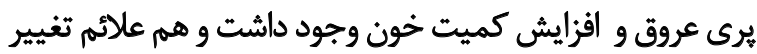

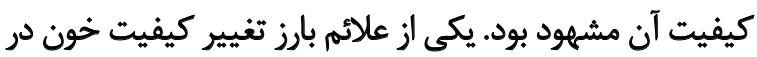

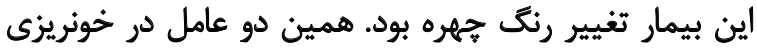

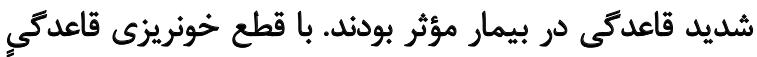

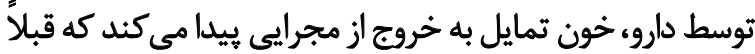

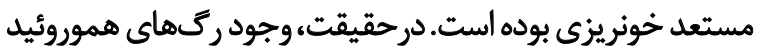

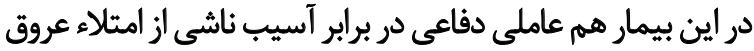

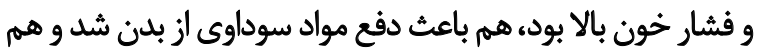

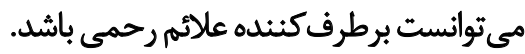

در اين مورد جه زيبا حكيم بوعلى سينا در كتاب وزين قانون

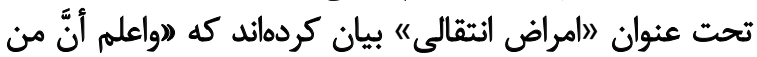

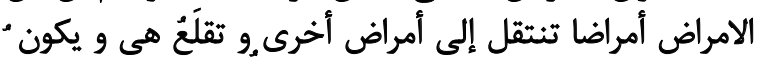

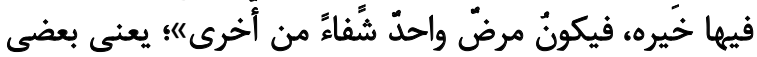

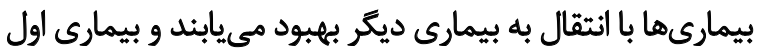

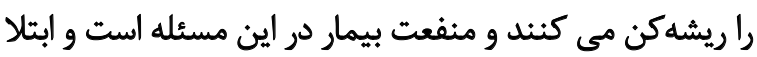

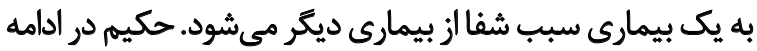

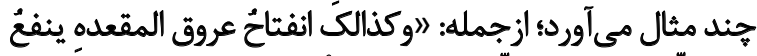

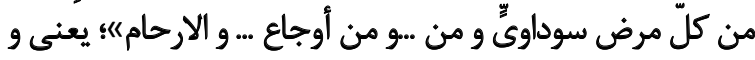

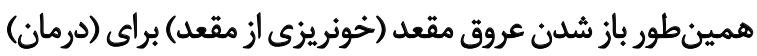

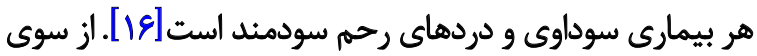

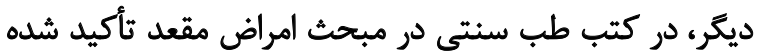

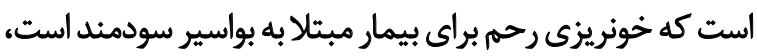

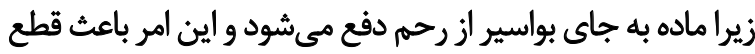

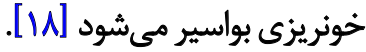

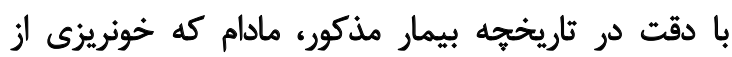

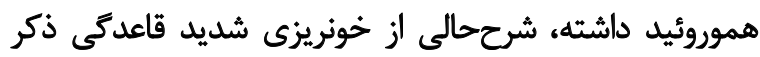

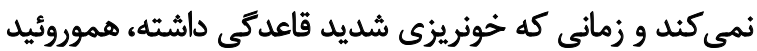

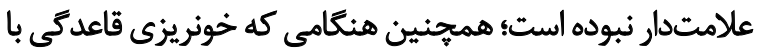

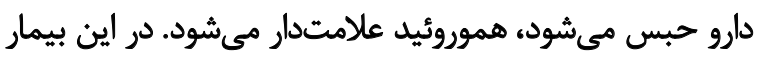

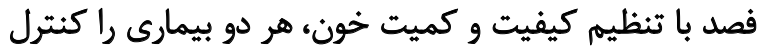

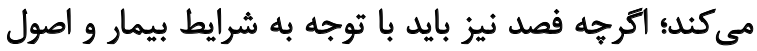

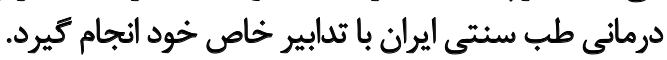

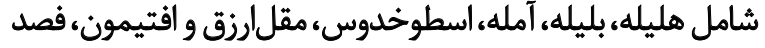

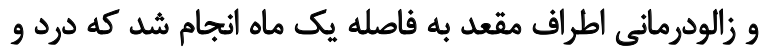

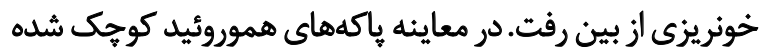

بودند.

ث

طب سنتى ايرانى هنكام برخورد با علائم بيمارى در اعضاى

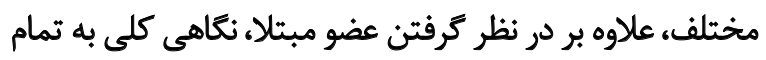

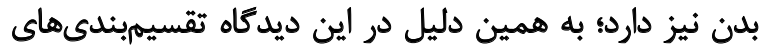

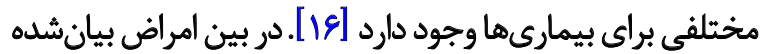

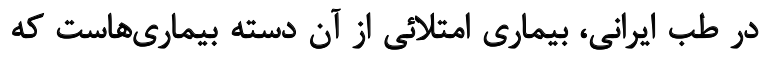

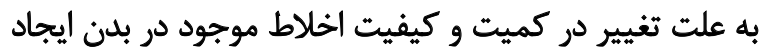

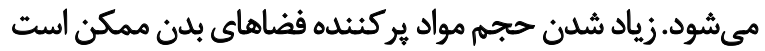

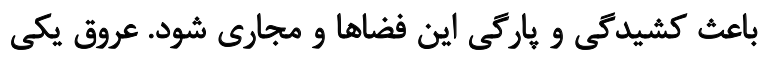

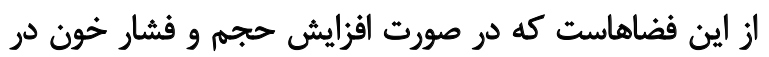

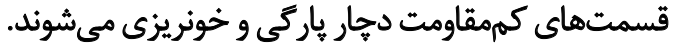
بر اساس طب ايرانى خون از تركيب هجهار خلط تشكيل شده

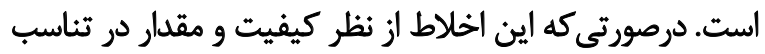

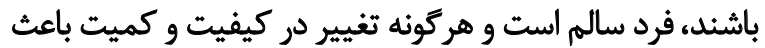

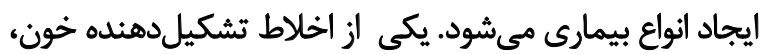

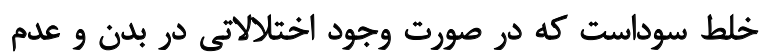
رعايت تدابير حفظ سلامتى، كيفيت آن تغيير مى كند. بر بر اساس

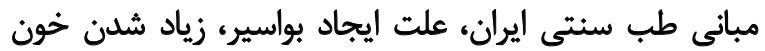

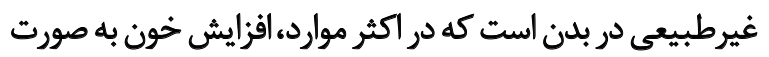

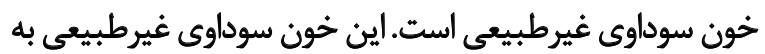

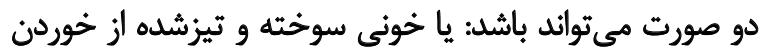

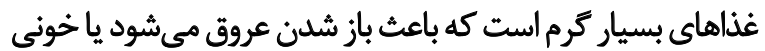

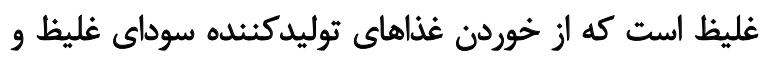
يرخورى و عدم هضم كامل غذا ايجاد شده است.

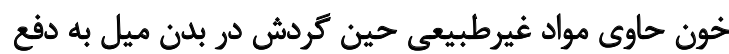

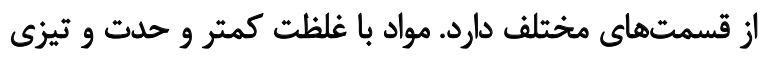

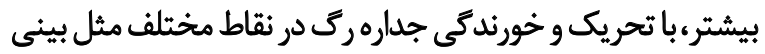

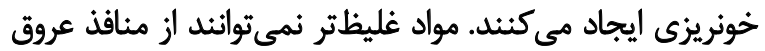

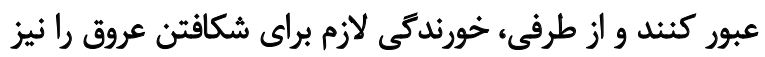

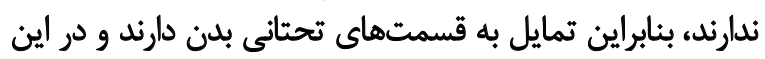

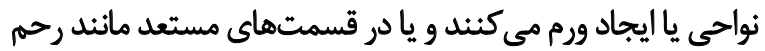

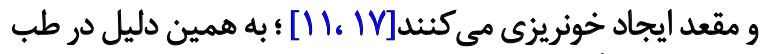

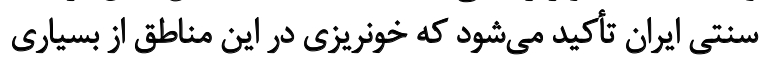

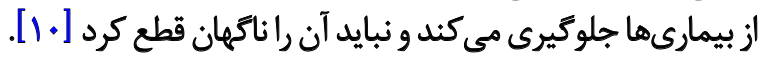

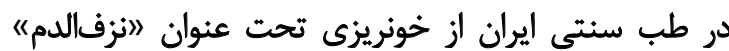

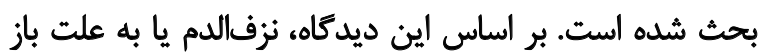

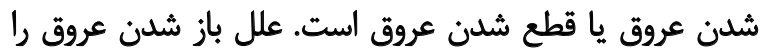

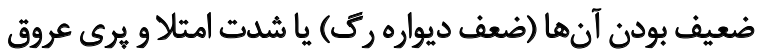


منافعى در خصوص يروهش حاضر وجود ندارد.

$$
\text { 1 }
$$

واز همكارى دانشكده طب ايرانى قم در انجام اين يثوهش تشكر

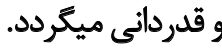

اين مطلب كه ناكنون گزارش موردى مشابه مورد كنونى

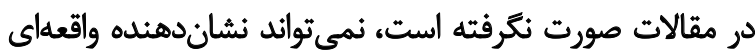

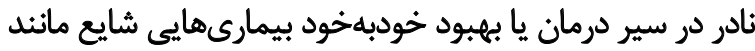

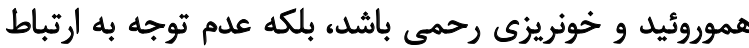

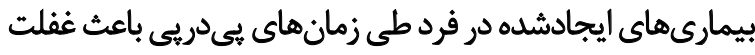

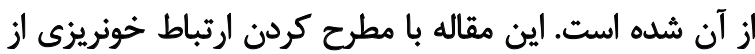

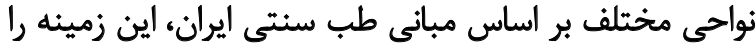

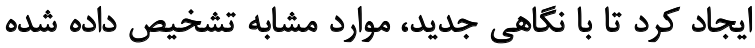

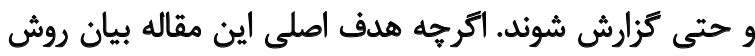

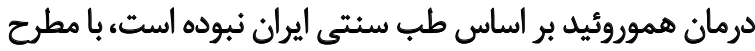

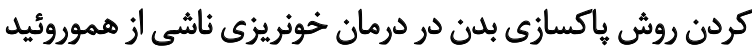

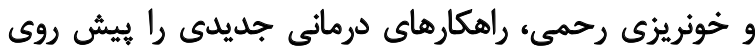

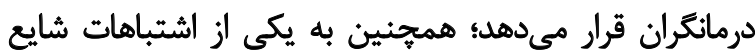

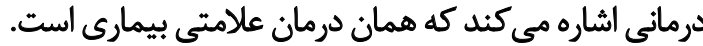

$$
\text { تثيجلميرى }
$$

در درمان بر اساس اصول طب ايرانى، برطرف كردن عامل اصلى إنى

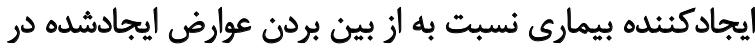

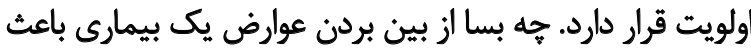

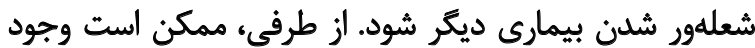

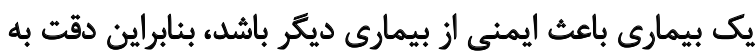

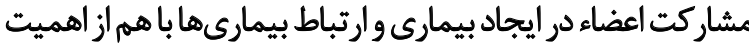

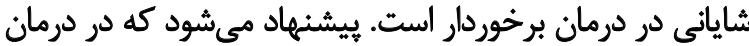

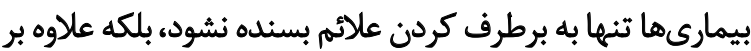

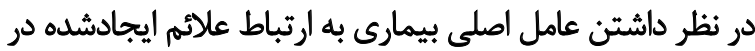
يك عضو با يجاد يا بيشكيرى از بيماري در اعضاء ديكر توجه شود.

مالاحظات اخلاقى

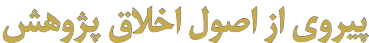

اين مطالعه باكد اخلاق IR.MUQ.REC.1396.110 در كميته اخلاق يُوهشى دانشكاه علوم يزشكى قم به تصويب رسيده است.

$$
\text { (l) }
$$

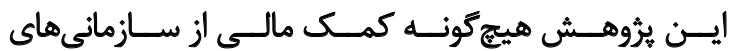

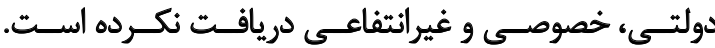

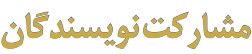

تمامى نويسندكان معيارهاى استاندارد نويسندكى بر اساس

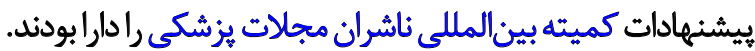

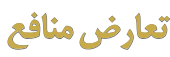

بدينوسيله نويسندكان تصريح مى كنند كه هيجّونه تضاد 


\section{References}

[1] Sandler RS, Peery AF. Rethinking what we know about hemorrhoids. Clin Gastroenterol Hepatol. 2019; 17(1):8-15. [DOI:10.1016/j. cgh.2018.03.020] [PMID] [PMCID]

[2] Riss S, Weiser FA, Schwameis K, Riss T, Mittlböck M, Steiner G, et al. The prevalence of hemorrhoids in adults. Int J Colorectal Dis. 2012; 27(2):215-20. [DOI:10.1007/s00384-011-1316-3] [PMID]

[3] Sun Z, Migaly J. Review of hemorrhoid disease: Presentation and management. Clin Colon Rectal Surg. 2016; 29(1):22-9. [DOI:10.1055/s-0035-1568144] [PMID] [PMCID]

[4] Margetis N. Pathophysiology of internal hemorrhoids. Ann Gastroenterol. 2019; 32(3):264-72. [DOI:10.20524/aog.2019.0355] [PMID] [PMCID]

[5] Strate L, Saltzman JR, Grover Sh. Approach to acute lower gastrointestinal bleeding in adults [Internet]. 2018 [Updated 2020 Apr 2]. Available from: https://www.uptodate.com/contents/approach-to-acute-lowergastrointestinal-bleeding-in-adults

[6] Mott T, Latimer K, Edwards C. Hemorrhoids: Diagnosis and treatment options. Am Fam Physician. 2018; 97(3):172-9. [PMID]

[7] Lohsiriwat V. Treatment of hemorrhoids: A coloproctologist's view. World J Gastroenterol. 2015; 21(31):9245-52. [DOI:10.3748/wjg.v21. i31.9245] [PMID] [PMCID]

[8] Avicenna. Canon of medicine (Al -Qanun- fi-al Tibb ). $3^{\text {rd }} \& 4^{\text {th }}$ ed. Beirut: Dar Al Ehya Al Toras Al Arabi; 2005.

[9] Nazem Jahan MA. Great Elixir. $3^{\text {rd }}$ ed. Tehran: Iran University of Medical Science; 2008.

[10] Arzani MA. [Explain the causes and signs in medicine (Persian)]. $1^{\text {st }}$ ed. Qom: Jalal-al-din; 2008.

[11] Jorjani E. Zakhireye Kharazmshahi. vol. 6. Qom: Tehran University of Medical Science; 2012.

[12] Rhazes M. Al-Havi. Khalife Taimi H, editor. vol. 11. Beirut: Dar Al Ehya Al Toras Al Arabi; 2001.

[13] Samarghandi N. Sharh al-Asbab va al-Alamat. vol. 2. Qom: Jalalal-din; 2008

[14] Akhawayni R. Hidāyat al Muta'allimin fi al-Tibb. $2^{\text {nd }}$ ed. Mashhad: Mashhad University of Medical Sciences; 1992.

[15] El-Nashar SA, Shazly SAM, Famuyide AO. Pictorial blood loss assessment chart for quantification of menstrual blood loss: A systematic review. Gynecol Surg. 2015; 12(3):157-63. [DOI:10.1007/ s10397-015-0893-5]

[16] Avicenna. Canon of medicine (Al-Qanun fi Tib). $1^{\text {st }}$ ed. Masoodi A, editor. Beirut: Dar Al Ehya Al Toras Al Arabi; 2013.

[17] Avicenna. Canon of medicin (Al-Qanun fi Tib). $2^{\text {nd }}$ ed. Masoodi A, editor. Tehran: Almai; 2013.

[18] Nafis G-I. Al-Mojaz fi tib. vol. 1. Beirut: Dar Al Mahagatolbayza; 2002. 